\title{
Atomic Spectral Tables for the Chandra X-ray Observatory
}

\author{
L. I. Podobedova, D. E. Kelleher, J. Reader, and W. L. Wiese \\ National Institute of Standards and Technology, Gaithersburg, MD \\ 20899-8420
}

\begin{abstract}
Tables of wavelengths, line classifications, and transition probabilities have been critically compiled for ionized spectra of neon (Ne V-VIII), magnesium (Mg V-X), silicon (Si VI-XII), and sulfur (S VIII-XIV) in the $20 \AA$ $170 \AA$ region. The tables provide basic atomic data for about 3300 transitions in support of astronomical studies from the Chandra X-ray Observatory.
\end{abstract}

\section{Introduction}

We have critically compiled spectral tables for higher ions of the cosmically abundant elements $\mathrm{Ne}, \mathrm{Mg}, \mathrm{Si}$, and $\mathrm{S}$ in the spectral range of interest for the Low Energy Transmission Grating (LETG) of the Chandra X-ray Observatory. Our tables which include 3300 transitions will also be of use for diagnostics of plasmas found in fusion energy research devices such as tokamaks.

The wavelengths in the tables are Ritz-type values derived from experimental energy level values in the NIST Atomic Spectra Database (1999) (ASD). That is, the wave number of a particular transition is found as the difference of the values of the combining energy levels in $\mathrm{cm}^{-1}$, and the wavelength in vacuum is the reciprocal of the wave number.

In compiling the transition probabilities we selected only values obtained with the most advanced theoretical and experimental methods. Our general evaluation criteria were those that have been developed at NIST (Wiese, Fuhr, \& Deters 1996). We normally list here only values having estimated uncertainties of $50 \%$ or less. A few exceptions have been made for important lines. Because of the very limited amount of experimental results available for highly ionized ions, for most transitions we had to rely on theoretical data.

The most extensive source of theoretical data was the Opacity Project (2002) (OP), which has produced multiplet $f$-values for the spectra of many elements. However, since the OP calculations do not include spin-orbit interaction, they do not provide values for individual lines of a fine-structure multiplet. Therefore for the present work the average values for $L S$ multiplets were decomposed into their $L S J$ fine structure components using $L S$ coupling rules. For the present light atoms $L S$ coupling should be a good approximation. For ions where this is clearly not the case we have used results of calculations that do include spin-orbit effects. Tachiev \& Froese Fischer (2002) have performed calculations for Be-, B-, C-, N-, O-, F-, and Ne-like ions with the multi-configuration HartreeFock (MCHF) method with Breit-Pauli corrections and have made their results available on the World Wide Web. Blackford \& Hibbert (1994) have carried out 
extensive calculations for F-like ions with the CIV3 code (CIV3 indicates Configuration Interaction code Version 3). The same method was used by Aggarwal (1998) for several C-like ions. Calculations with the many-body perturbation approach including Breit-Pauli corrections were also found to be very useful (Safronova et al. 1999; Vilkas et al. 1996). For comparative analysis data from several other sources were used in our work.

\section{Graphical and Numerical Comparisons in Support of the Assess- ment Procedure}

In order to reaffirm the uncertainty estimates of transition probabilities for the present compilation we made graphical and numerical comparisons of the results of different advanced calculations for as many transitions as possible, regardless of wavelength. Later we selected data for the Chandra spectral range $20-170$ $\AA$. To fit the data into systematic trends, or deviations from them, we found useful the theoretically predicted scaling of data along isoelectronic sequences. If available, we always selected data from detailed configuration-interaction calculations with intermediate coupling. As usual, these calculations are performed for transitions to the ground state or between low excited configurations. For the transitions involving high-lying configurations, only OP data are available. For the stronger transitions of many spectra, good agreement exists between the $\mathrm{OP}$ data and data from more detailed calculations that consider spin-orbit interactions.

The agreement among the OP calculations and different relativistic calculations gets worse for weaker transitions and for transitions between those levels where one or both are appreciably mixed due to breakdown of $L S$ coupling. Large disagreements are often observed for weaker transitions when calculations for the transition probabilities of weak transitions have encountered considerable problems due to appreciable cancellations of positive and negative components of the transition integral.

Problems concerning large discrepancies in transition probabilities for fluorine-like spectra between the OP and CIV3 (Blackford \& Hibbert 1994) results were recognized for some time and discussed earlier by Wiese \& Kelleher (1998). At that time extended relativistic calculations for individual lines were available only from CIV3 calculations. In recent years new MCHF data became available (Tachiev \& Froese Fischer 2002) which show that the agreement between MCHF and CIV3 is clearly better, but for many transitions it is still not good.

In studying the transitions for which the agreement is not good, we found that for almost all of them, one or both of the levels involved in the transition could be considered as mixed. By mixed we mean that the main contribution to the wave function of the level is less than $80 \%$. Correspondingly, a pure level here means that that the main contribution to the wave function composition of this level is more than $80 \%$.

Figure 1 shows a comparison of oscillator strengths of allowed transitions between mixed levels for the F-like ion S VIII. The ratios of CIV3 oscillator strengths (Blackford \& Hibbert 1994) to the corresponding MCHF values (Tachiev \& Froese Fischer 2002) are plotted on a logarithmic scale versus the MCHF oscillator strengths. The dashed lines indicate a band of $50 \%$ around a 


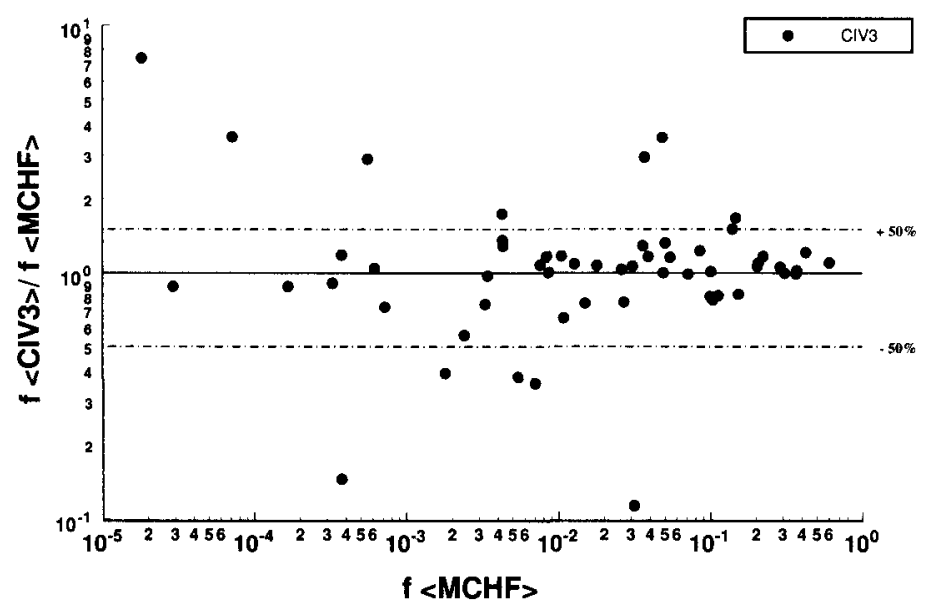

Figure 1. Comparison of oscillator strengths for S VIII for transitions with mixed levels 


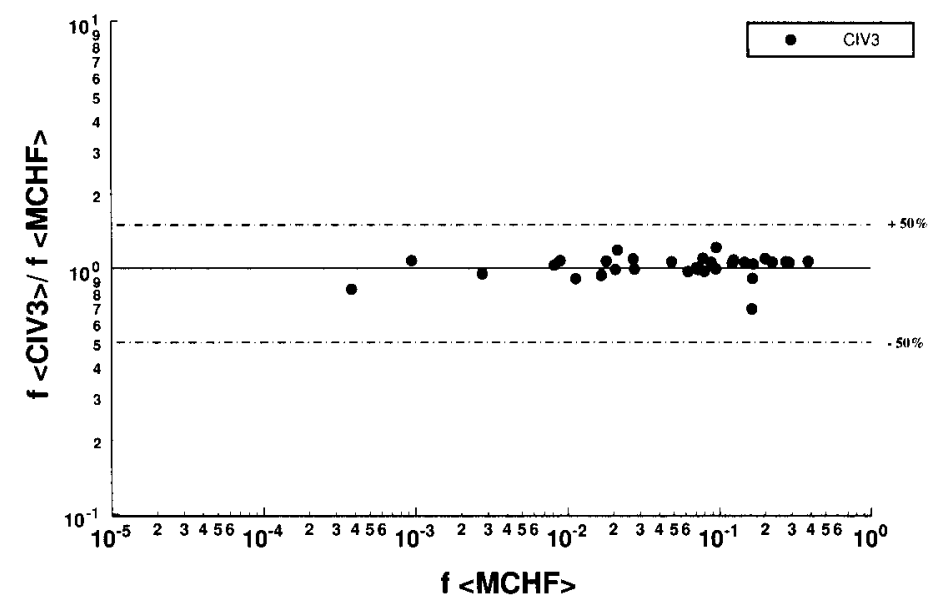

Figure 2. Comparison of oscillator strengths for S VIII for transitions with pure levels

perfect ratio of 1.00 . It is seen that for most "mixed" transitions the agreement is better than $50 \%$, which is within the range of data listed in the NIST reference tables.

For the "pure" transitions shown in Fig. 2 the agreement improves drastically. Out of 33 transitions, 31 have agreements between the CIV3 and MCHF calculations better than $10 \%$. The others agree within $20 \%$. The study of such cases is very valuable when we have transition probabilities available from only one source and we need to estimate their accuracy on the basis of extrapolations from comparisons with other sources in areas, where they overlap.

\section{Arrangement of the tables}

Atomic Spectral Data for the Chandra X-ray Observatory are presented by Podobedova et al (2003) on the world wide web. The tables are ordered for each chemical element by increasing ionization stage. Individual lines are arranged in order of wavelength, but otherwise in a format similar to the customary arrangement of the NIST ASD and the earlier NIST publications. Each transition is identified by its wavelength, the energy levels of the lower $(i)$ and upper $(k)$ states, the statistical weights of the levels $(g=2 J+1)$, and the level designations. All of the present values are for electric dipole transitions, E1. 
For each line, the transition probability for spontaneous emission $A_{k i}$ (in units of $10^{8} \mathrm{~s}^{-1}$ ), the oscillator strength $f_{i k}$ (dimensionless), and $\log g_{i} f$ are given. Also, the line strength $S$ is given and expressed in atomic units (a.u.). Finally, the estimated accuracy and the references are given. The estimated accuracy is indicated by the following letters, which are the same as used in earlier NIST references (see, e.g., Wiese et al. 1996):
A - uncertainty less than $3 \%$
B - uncertainty less than $10 \%$
C - uncertainty less than $25 \%$
D - uncertainty less than $50 \%$
E - uncertainty greater than $50 \%$ (but typically within factors of 2-3)

\section{Acknowledgments}

Support for this work was provided by the National Aeronautics and Space Administration through Chandra Award EL9-1002A issued by the Chandra X-Ray Observatory, which is operated by the Smithsonian Astrophysical Observatory for and on behalf of NASA under contract NAS8-39073. Partial support was also provided by the Office of Fusion Energy Sciences of the U. S. Department of Energy. Thanks are due to Jeffrey Fuhr for supplying neon data.

\section{References}

Aggarwal, K. M. 1998, ApJS, 118, 589

Blackford, H. M. S. \& Hibbert, A. 1994, ADNDT, 58, 101

NIST Atomic Spectra Database. 1999, URL http://atm.nist.gov/cgi-bin/AtData/main_asd/

Opacity Project. 2002, URL http://legacy.gsfc.nasa.gov/topbase

Podobedova, L. I., Musgrove A., Kelleher D. E., Reader J., Wiese W. L., Coursey J. S., \& Olsen, K. 2003,

URL http://physics.nist.gov/chandra

Safronova, U. I., Johnson, W. R., Safronova, M. S., \& Derevianko, A. 1999, Phys.Scr., 59,286 . Complete data listing from private communication.

Tachiev, G., \& Froese Fischer, C. 2002,

URL http://www.vuse.vanderbilt.edu/ $\sim$ cff/mchf_collection

Vilkas, M. J., Martinson, I., Merkelis, G., Gaigalas, G., \& Kisielius, R. 1996, Phys.Scr., 54,281

Wiese, W. L., Fuhr, J. R., \& Deters, T. M. 1996, JPCRD, Monograph 7, 532 pp.

Wiese, W. L. \& Kelleher, D. E. 1998 in AIP Conf. Proc., 434, Atomic and Molecular Data and Their Applications, ed. P. J. Mohr \& W. L. Wiese (New York: AIP), 105 